University of Nebraska - Lincoln

DigitalCommons@University of Nebraska - Lincoln

\title{
Consistent divergence times and allele sharing measured from cross-species application of SNP chips developed for three domestic species
}

\author{
J. M. Miller \\ University of Alberta, jmm1@ualberta.ca \\ J. W. Kijas \\ CSIRO Livestock Industries \\ M. P. Heaton \\ USDA-ARS, mike.heaton@usda.gov \\ J. C. Mcewan \\ AgResearch Invermay Agricultural Centre, john.mcewan@agresearch.co.nz \\ D. W. Coltman \\ University of Alberta
}

Follow this and additional works at: https://digitalcommons.unl.edu/hruskareports

Miller, J. M.; Kijas, J. W.; Heaton, M. P.; Mcewan, J. C.; and Coltman, D. W., "Consistent divergence times and allele sharing measured from cross-species application of SNP chips developed for three domestic species" (2012). Roman L. Hruska U.S. Meat Animal Research Center. 199.

https://digitalcommons.unl.edu/hruskareports/199

This Article is brought to you for free and open access by the U.S. Department of Agriculture: Agricultural Research Service, Lincoln, Nebraska at DigitalCommons@University of Nebraska - Lincoln. It has been accepted for inclusion in Roman L. Hruska U.S. Meat Animal Research Center by an authorized administrator of DigitalCommons@University of Nebraska - Lincoln. 


\title{
Consistent divergence times and allele sharing measured from cross-species application of SNP chips developed for three domestic species
}

\author{
J. M. MILLER,* J. W. KIJAS,+ M. P. HEATON, $\ddagger$ J. C. MCEWAN, $\S$ and D. W. COLTMAN* \\ *Department of Biological Sciences, University of Alberta, Edmonton, AB T6G 2E9, Canada, +CSIRO Livestock Industries, \\ St. Lucia, Qld 4067, Australia, łU.S. Meat Animal Research Center, USDA, ARS, Clay Center, NE 68933, USA, §AgResearch \\ Invermay Agricultural Centre, Mosgiel, 9053, New Zealand
}

\begin{abstract}
Recent advances in technology facilitated development of large sets of genetic markers for many taxa, though most often model or domestic organisms. Cross-species application of genomic technologies may allow for rapid marker discovery in wild relatives of taxa with well-developed resources. We investigated returns from cross-species application of three commercially available SNP chips (the OvineSNP50, BovineSNP50 and EquineSNP50 BeadChips) as a function of divergence time between the domestic source species and wild target species. Across all three chips, we observed a consistent linear decrease in call rate ( $1.5 \%$ per million years), while retention of polymorphisms showed an exponential decay. These results will allow researchers to predict the expected amplification rate and polymorphism of cross-species application for their taxa of interest, as well as provide a resource for estimating divergence times.
\end{abstract}

Keywords: marker development, phylogenomics, population genomics, SNP chip, wild taxa

Received 25 May 2012; revision received 10 August 2012; accepted 16 August 2012

\section{Introduction}

Marker development is the foundation from which all molecular ecology studies build. Techniques for marker discovery range from relatively low-throughput, targeted approaches (Palumbi 1996) to extremely highthroughput, multilocus methods (Baird et al. 2008; Morozova et al. 2009) which utilize next-generation sequencers. However, once a resource is developed for one species, it is often taken and applied to close relatives. For example, cross-species application of conserved primers has long been used to study mitochondrial DNA (Kocher et al. 1989), forming the basis for DNA barcoding (Hebert et al. 2003) and more recently metagenomic and environmental DNA analyses (Taberlet et al. 2012). Similarly, cross-species application has been widely used for microsatellite loci (e.g. Schlotterer et al. 1991; Primmer et al. 1996). Cross-species application of primers allows a researcher to skip development steps such as library construction and cloning, reducing costs and effort.

Correspondence: Joshua M. Miller, Fax: 1-780-492-9234;

E-mail: jmm1@ualberta.ca
Advancing sequencing technology has facilitated development of large panels of single nucleotide polymorphisms (SNPs) (Morin et al. 2004; Garvin et al. 2010). However, most of these marker sets are developed for model or domestic organisms and not for wildlife species. Development and application of large SNP sets is equally important in wild species where they would enable population genetic analyses writ large, examination of loci under selection, resolution of phylogenetic relationships and provide resources for conservation efforts (Stinchcombe \& Hoekstra 2008; Decker et al. 2009; Allendorf et al. 2010; Seeb et al. 2011; Angeloni et al. 2012). Cross-species application provides a potential method for rapid marker development if there are genomic resources developed for a closely related relative (Kohn et al. 2006). Several recent publications have applied commercially available SNP chips created for domestic animals to wild relatives (Pertoldi et al. 2010; Miller et al. 2011; Haynes \& Latch 2012; Ogden et al. 2012). However, all report a decrease in the number of loci that successfully amplify, and low retention of polymorphisms between species.

Here, we characterize the relationship between successful cross-species application of SNP chips and divergence time between the species for which the chip was 
made and the one to which it was applied. We compare results from three SNP chips developed for ungulates: the OvineSNP50 BeadChip (Kijas et al. 2012), the BovineSNP50 BeadChip (Matukumalli et al. 2009) and the EquineSNP50 BeadChip (McCue et al. 2012). Based on predictions from the neutral theory of molecular evolution (Kimura \& Ohta 1969; Ohta 1992) and the binding dynamics associated with genotyping via oligonucleotide arrays (Sechi et al. 2010), we expected to observe slow declines in call rate as a function of divergence time, and a much more rapid decrease in retention of polymorphism.

\section{Methods}

\section{Data sets}

Development and genotyping using the OvineSNP50 BeadChip are described in the study by Kijas et al. (2012) and Miller et al. (2011). DNA from wild sheep and outgroup species (Table S1, Supporting information) was genotyped using the same methods as described for domestic sheep (Kijas et al. 2012). Raw intensity data were converted into genotype calls using GenomeStudio, before genotypes with GT score lower than 0.8 were removed. All subsequent analysis of the SNP file was conducted with PLINK (Purcell et al. 2007). We extracted loci which were successfully amplified in a given species ( $>50 \%$ of individuals typed), including those mapped to the $X$ chromosome in domestic sheep. We then determined the number of polymorphic loci, that is, those with a minor allele frequency $\geq 1 \%$. Data for the BovineSNP50 and EquineSNP50 arrays were collected from previous publications (Table S1, Supporting information). If multiple quality filtering metrics were presented for the same species, we retained only data corresponding to the most stringent criteria.

\section{Analyses}

We first considered the percent of markers on a chip that were called for each wild species. Note that in two cases (Pertoldi et al. 2010; Haynes \& Latch 2012), call rates were presented as averages over all species examined in the respective studies ( $N=3$ for each), all other estimates were species-specific. We then conducted weighted regression of call rate against time to last common ancestor (LCA) for each chip type individually. Weights corresponded to the number of individuals of each taxon that were typed. Estimates of time to LCA came either from the text of the individual studies, or from the studies by Fernandez \& Vrba (2005); Hiendleder et al. (2008); Steiner \& Ryder (2011). Lastly, we assessed whether the relationships differed between chips using ANCOVA, fitting an interaction term among chip type and time to LCA.

To assess retention rate of polymorphic loci, we conducted a single weighted regression of percentage of polymorphic loci (as a function of those called for a species) against time to LCA for all chips pooled using a fitted curve. Samples were pooled given that there were no differences in the call rate among arrays (see Results). All regressions and curve fitting were carried out using R v2.13.0.

\section{Results}

The OvineSNP50 Beadchip was applied to 16 wild taxa, and genotyping wild sheep revealed very high call rates $(>98 \%)$ for species such as bighorn, argali and urial sheep that have LCA c. 2.4 MYA or less. The most distantly related species tested using the OvineSNP50 Beadchip (pronghorn antelope; LCA 33.2 MYA) revealed a call rate of $40.8 \%$. These data were combined with six published studies that applied the BovineSNP50 to 74 taxa and EquineSNP50 to 17 taxa (see Table S1, Supporting information). All three chips showed linear declines in observed call rate with increasing time to last common ancestor (Fig. 1, Table 1). There were no significant differences in slope between the three chips (ANCOVA interaction between time and chip type: $F_{2,99}=0.6173$, $P=0.5415)$. Polymorphism data were not available for eight taxa on the equine array. The relationship between polymorphism and time to LCA for the remaining 94

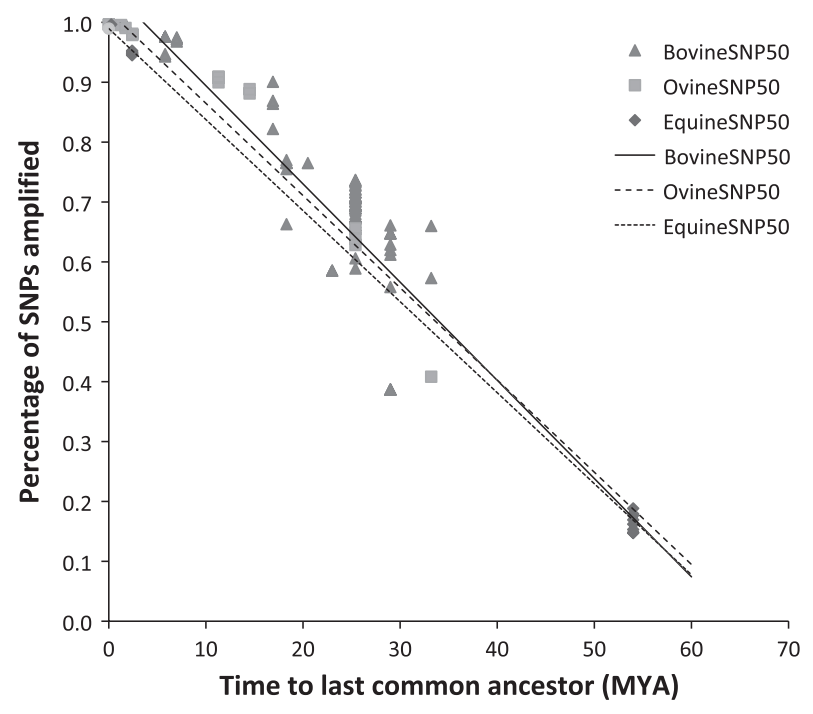

Fig. 1 Scatter plot of call rate (per cent) as a function of time to last common ancestor for each SNP chip examined in this study. Weighted regression lines are shown. MYA is millions of years ago. 
Table 1 Coefficients describing the relationship between call rate and time to last common ancestor for the three SNP arrays. All values are highly significant $(P<<0.0001)$

\begin{tabular}{lllll}
\hline Chip & Slope & SE & Intercept & SE \\
\hline BovineSNP50 & -0.0164 & 0.0008 & 1.0582 & 0.0154 \\
OvineSNP50 & -0.0154 & 0.0011 & 1.0188 & 0.0128 \\
EquineSNP50 & -0.0152 & 0.0001 & 0.9896 & 0.0034 \\
\hline
\end{tabular}

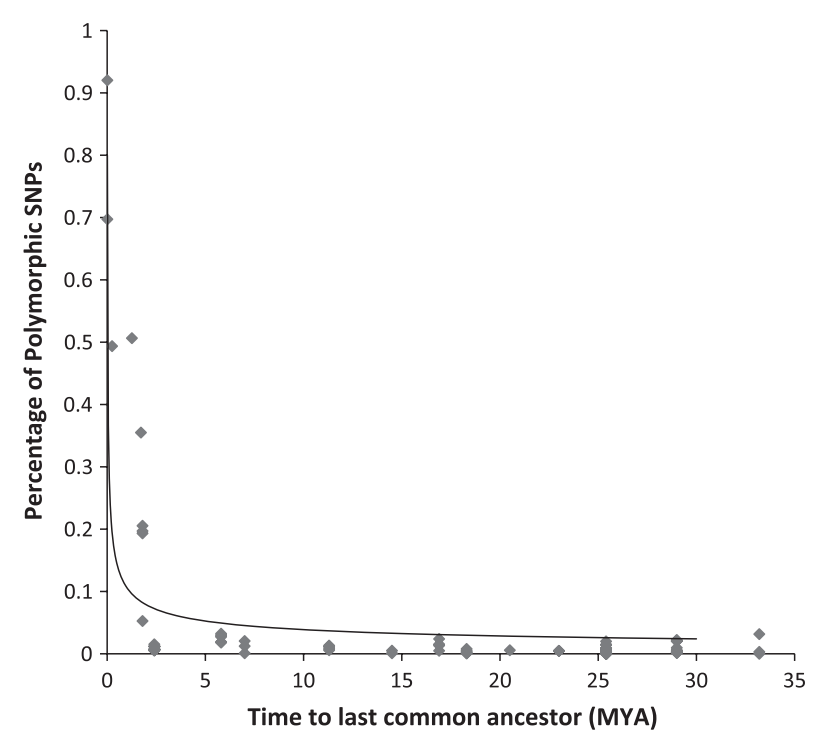

Fig. 2 Scatter plot of percentage of amplified loci which retained polymorphism as a function of time to last common ancestor for all SNP chips examined in this study. A nonlinear weighted regression line is shown. MYA is millions of years ago.

taxa was best described by an exponential decay function of $y=0.1063 x^{-0.4382}-0.02$ (Fig. 2).

\section{Discussion}

Cross-species call rate decreases by about $1.5 \%$ with each million-year divergence between the species for which a chip was developed and the species it was applied to. This consistent pattern suggests it would be possible to gauge expected success of application of these chips in taxa of interest before investing in the costs of genotyping and accurately estimate divergence times between taxa. Linear decreases in call rate are consistent with expectations of neutral sequence evolution and heteroduplex formation between an oligonucleotide probe and genomic DNA. Specifically, hybridization is prevented if there are mutations in the flanking sequence of the SNP (Sechi et al. 2010). The accumulation of polymorphisms in flanking sequence should fit basic neutral substitution models (Kimura 1968; Ohta 1992) that are dependent on mutation rate, but linear with respect to time.

There was a much more drastic decline in percentage of loci that retained polymorphisms as a function of time to LCA. Low levels of shared polymorphisms are consistent with theoretical expectations as well. The retention or fixation of a SNP is expected to have a logarithmic decline at a rate that is dependent on population size as well as mutation rate (Kimura \& Ohta 1969; Ohta 1992). These steep rates of decline may be exacerbated by the use of novel or rare alleles that arose as a result of the domestication process. Our regression indicates that 50\% of loci will remain polymorphic only when species are less than c. 30000 years divergent, and application of a chip to species which diverged 3 MYA from the species it was intended for will have only $\sim 5 \%$ of amplified loci remain polymorphic. It is interesting to note that the percentage of polymorphic SNPs appears to level off after c. $5 \mathrm{Myr}$ divergence. Some level of shared polymorphism is to be expected; however, these shared sites are unlikely to be the result of retention of the same mutation because of selective advantage (Asthana et al. 2005) but more likely de novo mutations (Nowell et al. 2011).

Most of the SNPs discovered via commercial SNP chips are expected to be selectively neutral. For example, $65 \%$ of SNPs on the OvineSNP50 BeadChip are thought to be intergenic and not within $2.5 \mathrm{~kb}$ of a gene as loci were chosen to achieve approximately even spacing of SNPs across the sheep genome. This pattern is likely to be true for the other chips as well, where loci were also chosen based on spacing throughout the genome. However, an underlying issue with cross-species application of SNP arrays is that the SNPs discovered may be enriched for 'older' shared polymorphisms, and rare or novel variants are missed, introducing some level of ascertainment bias. However, ascertainment bias is inherent in any marker development (Clark et al. 2005; Rosenblum \& Novembre 2007) and can be compensated for. Prior to formal analysis, loci can be subjected to preliminary screening, such as for deviation from neutrality or examining the distribution of minor allele frequencies, and removed or retained according to the focus of the study at hand. Alternatively, the discovery scheme can be directly factored into the analysis using various algorithms or models that can assess and correct the underlying allele frequency spectrum (e.g. Nielsen \& Signorovitch 2003).

As more chips continue to be produced, it will be interesting to investigate what other factors may influence the patterns we observed, such as composition of the original SNP discovery panel or the intended goal of the chip. Although the three chips examined here had different discovery panels both in terms of number of individuals and SNP sources (Matukumalli et al. 2009; 
Kijas et al. 2012; McCue et al. 2012), they were developed with similar goals in mind: broadly, breed discrimination and assisting in targeted trait development through genome-wide association studies. Chips developed using data from multiple breeds or those based on species with different evolutionary histories may lead to different conversion rates (Satkoski Trask et al. 2011). An interesting point of comparison will come when chips derived from, and intended for, wild species are available and then applied to other wild relatives. A 'wild' SNP chip may have a larger amount of standing genetic variation to pull from during development, and without the history of artificial selection, polymorphisms may be more likely to be held in common across taxa.

We assessed the transferability of our results for nonungulates using data from two additional studies (Hacia et al. 1999; vonHoldt et al. 2011). Expected returns very closely parallel the observed results (Table S2, Supporting information), with the exception of higher than expected proportion of polymorphism retention in wolves and coyotes. However, this may be due to continuous interbreeding with domestic dogs, and inclusion of those taxa in the discovery panel (vonHoldt et al. 2011).

An alternative to cross-species application of SNP chips for marker discovery and genotyping are various genotype-by-sequencing (GBS) approaches (Davey et al. 2011; Elshire et al. 2011). These methods are likely to discover a multitude of species-specific SNPs, and avoid ascertainment bias caused by enriching for variants shared across species (though, rare SNPs are still likely to be underrepresented). However, there are some significant limitations when compared with SNP chips. First, error rates are likely to be higher with GBS than SNP chips. GBS approaches rely on pooling multiple individuals that have been labelled with unique barcodes into a single run on a next-generation sequencer. Sequencing reads are then partitioned across individuals and loci, which may result in low coverage for some individual/ locus combination and hence genotyping errors, especially, as the number of individuals or loci increases. Sequencing each locus to a greater depth would prevent this problem, but that is only accomplished either by purchasing more time on a next-generation sequencer or by reducing the number of individual or loci examined. SNP chips, on the other hand, have built in redundancy where each locus is present multiple times, and a genotype is called by averaging over all of the individual calls leading to accurate genotypes (Oliphant et al. 2002; Steemers \& Gunderson 2007). Second, it is more difficult to get a core set of loci genotyped in every individual. This is due to the fact that many GBS methods involve digestion with restriction enzymes that can lead to high variance between individuals on which loci are present in the sequencing library. Some of these limitations can be overcome by imputation of genotypes (Li et al. 2009; Pasaniuc et al. 2012), provided a reference panel of genotypes is available. In contrast, the same loci will always be present on a SNP chip. Lastly, the relative costs of each method also need to be considered, but these may vary depending on the application and questions addressed in a particular study. These limitations are further exacerbated if one wants to consider multiple species, which will hamper the use of GBS methods in phylogenetic analyses where having homologous regions of the genome is of the utmost importance (McCormack et al. 2012, in press).

In many cases, even if only $1 \%$ of loci on a $50 \mathrm{k}$ SNP chip are polymorphic, those 500 loci would represent a substantial increase in genetic resources available for a wild species. Beyond marker discovery, call rate decrease from Illumina technologies can provide a way to rapidly estimate the divergence time and evolutionary relationship among poorly studied species. In addition to the chips examined here, porcine (Ramos et al. 2009), chicken (Groenen et al. 2011) as well as various murine and human arrays are available. Thus, these technologies can provide resources in a vast and diverse array of wild species.

\section{Acknowledgements}

JMM is supported by a graduate research scholarship from Alberta Innovates Technologies Future and is a Natural Sciences and Engineering Research Council of Canada (NSERC) Vanier scholar. DWC is also funded by NSERC. We would also like to thank Dr. Shawn Narum and two anonymous reviewers for constructive comments on earlier versions of the manuscript.

\section{References}

Allendorf FW, Hohenlohe PA, Luikart G (2010) Genomics and the future of conservation genetics. Nature Review Genetics, 11, 697-709.

Angeloni F, Wagemaker N, Vergeer P, Ouborg J (2012) Genomic toolboxes for conservation biologists. Evolutionary Applications, 5, 130-143.

Asthana S, Schmidt S, Sunyaev S (2005) A limited role for balancing selection. Trends in Genetics, 21, 30-32.

Baird NA, Etter PD, Atwood TS, et al. (2008) Rapid SNP discovery and genetic mapping using sequenced RAD markers. PLOS ONE, 3, e3376.

Clark AG, Hubisz MJ, Bustamante CD, Williamson SH, Nielsen R (2005) Ascertainment bias in studies of human genome-wide polymorphism. Genome Research, 15, 1496-1502.

Davey JW, Hohenlohe PA, Etter PD, Boone JQ, Catchen JM, Blaxter ML (2011) Genome-wide genetic marker discovery and genotyping using next-generation sequencing. Nature Reviews Genetics, 12, 499-510.

Decker J, Pires J, Conant G et al. (2009) Resolving the evolution of extant and extinct ruminants with high-throughput phylogenomics. Proceedings of the National Academy of Sciences of the United States of America, 106, 18644-18649.

Elshire R, Glaubitz J, Sun Q et al. (2011) A robust, simple genotypingby-sequencing (GBS) approach for high diversity species. PLoS ONE, 6, doi:10.1371/journal.pone.0019379. 
Fernandez M, Vrba E (2005) A complete estimate of the phylogenetic relationships in Ruminantia: a dated species-level supertree of the extant ruminants. Biological Reviews of the Cambridge Philosophical Society, 80, 269-302.

Garvin MR, Saitoh K, Gharrett AJ (2010) Application of single nucleotide polymorphisms to non-model species: a technical review. Molecular Ecology Resources, 10, 915-934.

Groenen M, Megens H-J, Zare Y et al. (2011) The development and characterization of a $60 \mathrm{~K}$ SNP chip for chicken. BMC Genomics, 12, doi:10.1186/1471-2164-1112-1274.

Hacia J, Fan J, Ryder O et al. (1999) Determination of ancestral alleles for human single-nucleotide polymorphisms using high-density oligonucleotide arrays. Nature Genetics, 22, 164-167.

Haynes G, Latch E (2012) Identification of Novel Single Nucleotide Polymorphisms (SNPs) in Deer (Odocoileus spp.) using the bovinesnp50 beadchip. PLoS ONE, 7, e36536.

Hebert PDN, Cywinska A, Ball SL, DeWaard JR (2003) Biological identifications through DNA barcodes. Proceedings of the Royal Society of London Series B-Biological Sciences, 270, 313-321.

Hiendleder S, Lewalski H, Janke A (2008) Complete mitochondrial genomes of Bos taurus and Bos indicus provide new insights into intraspecies variation, taxonomy and domestication. Cytogenetic and Genome Research, 120, 150-156.

vonHoldt BM, Pollinger JP, Earl DA et al. (2011) A genome-wide perspective on the evolutionary history of enigmatic wolf-like canids. Genome Research, 21, 1294-1305.

Kijas JW, Lenstra JA, Hayes B et al. (2012) Genome-wide analysis of the world's sheep breeds reveals high levels of historic mixture and strong recent selection. PLoS Biology, 10, e1001258.

Kimura M (1968) Evolutionary rate at the molecular level. Nature, 217, 624-626.

Kimura M, Ohta T (1969) The average number of generations until extinction of an individual mutant gene in a finite population. Genetics, 63, 701-709.

Kocher T, Thomas W, Meyer A et al. (1989) Dynamics of mitochondrial DNA evolution in animals: amplification and sequencing with conserved primers. Proceedings of the National Academy of Sciences, 86, 6196-6200.

Kohn MH, Murphy WJ, Ostrander EA, Wayne RK (2006) Genomics and conservation genetics. Trends in Ecology \& Evolution, 21, 629637.

Li Y, Willer C, Sanna S, Abecasis G (2009) Genotype Imputation. Annual Review of Genomics and Human Genetics, 10, 387-406.

Matukumalli L, Lawley C, Schnabel R et al. (2009) Development and characterization of a high density SNP genotyping assay for cattle. PLoS ONE, 4, doi:10.1371/journal.pone.0005350.

McCormack J, Maley J, Hird S, Derryberry EP, Graves GR, Brumfield RT (2012) Next-generation sequencing reveals phylogeographic structure and a species tree for recent bird divergences. Molecular Phylogenetics and Evolution, 62, 397-406.

McCormack JE, Hird SM, Zellmer AJ, Carstens BC, Brumfield RT (In press) Applications of next-generation sequencing to phylogeography and phylogenetics. Molecular Phylogenetics and Evolution, doi:10.1016/j. ympev.2011.12.007.

McCue ME, Bannasch DL, Petersen JL et al. (2012) A high density SNP array for the domestic horse and extant Perissodactyla: utility for association mapping, genetic diversity, and phylogeny studies. PLoS Genetics, 8, e1002451.

Miller JM, Poissant J, Kijas J, Coltman DW, The International Sheep Genomics Consortium (2011) A genome-wide set of SNPs detects population substructure and long range linkage disequilibrium in wild sheep. Molecular Ecology Resources, 11, 314-322.

Morin PA, Luikart G, Wayne RK, Grp SNPW (2004) SNPs in ecology, evolution and conservation. Trends in Ecology \& Evolution, 19, 208-216.

Morozova O, Hirst M, Marra MA (2009) Applications of new sequencing technologies for transcriptome analysis. Annual Review of Genomics and Human Genetics, 10, 135-151.
Nielsen R, Signorovitch J (2003) Correcting for ascertainment biases when analyzing SNP data: applications to the estimation of linkage disequilibrium. Theoretical Population Biology, 63, 245-255.

Nowell R, Charlesworth B, Haddrill P (2011) Ancestral polymorphisms in Drosophila pseudoobscura and Drosophila miranda. Genetics Research, 93, 255-263.

Ogden R, Baird J, Senn H, McEwing R (2012) The use of cross-species genome-wide arrays to discover SNP markers for conservation genetics: a case study from Arabian and scimitar-horned oryx. Conservation Genetics Resources, 4, 471-473.

Ohta T (1992) The nearly neutral theory of molecular evolution. Annual Review of Ecology and Systematics, 23, 263-286.

Oliphant A, Barker D, Stuelpnagel J, Chee M (2002) BeadArray technology: enabling an accurate, cost-effective approach to high-throughput genotyping. BioTechniques, 32, S56.

Palumbi SR (1996) Nucleic acids II: the polymerase chain reaction. In: Molecular Systematics (eds Hillis DM, Moritz C \& Mable BK), pp. 205-221. Sinauer Associates Inc., Sunderland, Massachusetts.

Pasaniuc B, Rohland N, McLaren P et al. (2012) Extremely low-coverage sequencing and imputation increases power for genome-wide association studies. Nature Genetics, 44, 631-U641.

Pertoldi C, Wójcik J, Tokarska M et al. (2010) Genome variability in European and American bison detected using the BovineSNP50 BeadChip. Conservation Genetics, 11, 627-634.

Primmer C, Moller A, Ellegren H (1996) A wide-range survey of crossspecies microsatellite amplification in birds. Molecular Ecology, 5, 365378.

Purcell S, Neale B, Todd-Brown K et al. (2007) PLINK: a tool set for whole-genome association and population-based linkage analyses. American Journal of Human Genetics, 81, 559-575.

Ramos A, Crooijmans R, Affara N et al. (2009) Design of a high density SNP genotyping assay in the pig using SNPs identified and characterized by next generation sequencing technology. PLoS ONE, 4, doi:10.1371/journal.pone.0006524.

Rosenblum EB, Novembre J (2007) Ascertainment bias in spatially structured populations: a case study in the Eastern fence lizard. Journal of Heredity, 98, 331-336.

Satkoski Trask J, Malhi R, Kanthaswamy S et al. (2011) The effect of SNP discovery method and sample size on estimation of population genetic data for Chinese and Indian rhesus macaques (Macaca mulatta). Primates, 52, 129-138.

Schlotterer C, Amos B, Tautz D (1991) Conservation of polymorphic simple sequence loci in cetacean species. Nature, 354, 63-65.

Sechi T, Coltman DW, Kijas JW (2010) Evaluation of 16 loci to examine the cross-species utility of single nucleotide polymorphism arrays. Animal Genetics, 41, 199-202.

Seeb J, Carvalho G, Hauser L et al. (2011) Single-nucleotide polymorphism (SNP) discovery and applications of SNP genotyping in nonmodel organisms. Molecular Ecology Resources, 11, 1-8.

Steemers FJ, Gunderson KL (2007) Whole genome genotyping technologies on the BeadArray ${ }^{\mathrm{TM}}$ platform. Biotechnology Journal, 2, 41-49.

Steiner C, Ryder O (2011) Molecular phylogeny and evolution of the Perissodactyla. Zoological Journal of the Linnean Society, 163, 1289-1303.

Stinchcombe JR, Hoekstra HE (2008) Combining population genomics and quantitative genetics: finding the genes underlying ecologically important traits. Heredity, 100, 158-170.

Taberlet P, Coissac E, Pompanon F, Brochmann C, Willerslev E (2012) Towards next-generation biodiversity assessment using DNA metabarcoding. Molecular Ecology, 21, 2045-2050.

J.M.M. conceived the study, conducted data analyses and drafted the original manuscript. J.W.K., M.P.H., J.C. M., and D.W.C. provided DNA samples and funded the collection of SNP genotypes using the OvineSNP50 BeadChip. All authors contributed analytical guidance 
and provided input to the manuscript throughout it's preparation.

\section{Data Accessibility}

All data used in this study can be found in the Tables S1 and S2 (Supporting information).

\section{Supporting Information}

Additional Supporting Information may be found in the online version of this article:
Table S1 List of species examined with their associated amplification success, percentage of polymorphic loci, and estimate of divergence time.

Table S2 List of taxa used to assess transferability of our results with their associated amplification success, percentage of polymorphic loci, and estimate of divergence time.

Please note: Wiley-Blackwell are not responsible for the content or functionality of any supporting materials supplied by the authors. Any queries (other than missing material) should be directed to the corresponding author for the article. 